\title{
Hot Electrons, Hot Holes, or Both? Tandem Synthesis of Imines Driven by the Plasmonic Excitation in $\mathrm{Au} / \mathrm{CeO}_{2}$ Nanorods
}

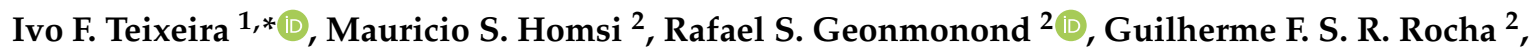

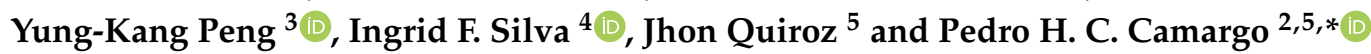 \\ 1 Department of Chemistry, Federal University of São Carlos, São Carlos 13565-905, Brazil \\ 2 Department of Fundamental Chemistry, Institute of Chemistry, University of São Paulo, São Paulo 05508-000, \\ Brazil; mauriciosamuel.homsi@usp.br (M.S.H.); rafaelsg31@gmail.com (R.S.G.); \\ guilhermefrrocha@gmail.com (G.F.S.R.R.) \\ 3 Department of Chemistry, City University of Hong Kong, Yeung Kin Man Academic Building, Hong Kong, \\ China; ykpeng@cityu.edu.hk \\ 4 Department of Chemistry, ICEx, Universidade Federal de Minas Gerais, Belo Horizonte 31270-901, Brazil; \\ ingridfs@ufmg.br \\ 5 Department of Chemistry, University of Helsinki, A.I. Virtasen aukio 1, 00100 Helsinki, Finland; \\ jhon.quiroz@helsinki.fi \\ * Correspondence: ivo@ufscar.br (I.F.T.); pedro.camargo@helsinki.fi (P.H.C.C.)
}

Received: 15 July 2020; Accepted: 31 July 2020; Published: 4 August 2020

\begin{abstract}
Solar-to-chemical conversion via photocatalysis is of paramount importance for a sustainable future. Thus, investigating the synergistic effects promoted by light in photocatalytic reactions is crucial. The tandem oxidative coupling of alcohols and amines is an attractive route to synthesize imines. Here, we unravel the performance and underlying reaction pathway in the visible-light-driven tandem oxidative coupling of benzyl alcohol and aniline employing $\mathrm{Au} / \mathrm{CeO}_{2}$ nanorods as catalysts. We propose an alternative reaction pathway for this transformation that leads to improved efficiencies relative to individual $\mathrm{CeO}_{2}$ nanorods, in which the localized surface plasmon resonance (LSPR) excitation in Au nanoparticles (NPs) plays an important role. Our data suggests a synergism between the hot electrons and holes generated from the LSPR excitation in Au NPs. While the oxygen vacancies in $\mathrm{CeO}_{2}$ nanorods trap the hot electrons and facilitate their transfer to adsorbed $\mathrm{O}_{2}$ at surface vacancy sites, the hot holes in the Au NPs facilitate the $\alpha-\mathrm{H}$ abstraction from the adsorbed benzyl alcohol, evolving into benzaldehyde, which then couples with aniline in the next step to yield the corresponding imine. Finally, cerium-coordinated superoxide species abstract hydrogen from the Au surface, regenerating the catalyst surface.
\end{abstract}

Keywords: tandem; oxidative coupling; $\mathrm{Au} \mathrm{NPs} ; \mathrm{CeO}_{2}$ nanorods; localized surface plasmon resonance; nanocatalysis

\section{Introduction}

Photocatalysis can initiate or accelerate chemical reactions by the light-matter interaction, which can simultaneously solve the problems about solar energy conversion and storage [1]. Photocatalysts can directly convert solar energy into chemical energy and simultaneously accomplish solar energy conversion and storage objectives [1]. Metal/semiconductor hybrid photocatalysts have emerged as one of the most promising catalytic systems to promote solar-to-chemical conversion. Therefore investigation of the synergistic effects promoted by light in these hybrid photocatalytic systems is of paramount importance for the design of new photocatalysts containing plasmonic 
components that can enable improved performance and selectivity via reaction pathways that are not accessible in semiconducting nanoparticles (NPs) or external heating.

The tandem conversion of alcohols into imines, via alcohol oxidation followed by an amine coupling, is of great interest in organic and green chemistry as it can be carried out under mild conditions, alcohols are widely available, and only hydrogen or water are generated as byproducts [2-6]. $\mathrm{CeO}_{2} \mathrm{NPs}$ have been effective as catalysts towards this tandem transformation, where a correlation between oxygen vacancies (as enabled by distinct nanoparticle shapes) and the catalytic activity has been established [6-8]. For instance, $\mathrm{CeO}_{2}$ nanorods have shown improved performance relative to nanocubes and nanoctahedra due to the exposure of (110) surface facets that enable a greater concentration of oxygen vacancies $[7,8]$.

It has been shown that the nonradiative decay following localized surface plasmon resonance (LSPR) excitation in plasmonic metals leads to hot electrons and holes, that can participate in the electronic or vibrational activation of species close to the surface $[9,10]$. This provides sufficient energy to initiate, accelerate, and/or control molecular transformations $[9,10]$. For metals such as $\mathrm{Ag}, \mathrm{Au}, \mathrm{Al}$, and $\mathrm{Cu}$, the LSPR excitation can take place in the visible range [11,12]. Interestingly, the combination of $\mathrm{CeO}_{2}$ and plasmonic NPs, such as $\mathrm{Au}$, can provide opportunities for not only achieving improved performance but also opening up reaction pathways under light-excitation that are not accessible via external heating [13-18].

In this work, we unravel the performance and underlying reaction pathway in the visible-light-driven tandem oxidative coupling of benzyl alcohol and aniline employing $\mathrm{Au} / \mathrm{CeO}_{2}$ nanorods as catalysts. We propose an alternative reaction pathway for this transformation that leads to improved efficiencies relative to individual $\mathrm{CeO}_{2}$ nanorods, in which the LSPR excitation in $\mathrm{Au}$ NPs played an important role. Our data suggests a synergism between the hot electrons and holes generated from the LSPR excitation in Au NPs, which acted in combination with the oxygen vacancies in the $\mathrm{CeO}_{2}$ to improve catalytic activities.

\section{Experimental Section}

\subsection{Au Nanoparticles Synthesis}

Spherical gold nanoparticles with 10-20 nm diameter were synthesized using $\mathrm{HAuCl}_{4}$, deionized water, ascorbic acid and polyvinylpyrrolidone (PVP). To a $50 \mathrm{~mL}$ round bottom flask were added $6 \mathrm{~mL}$ deionized water, $35 \mathrm{mg}$ PVP and $60 \mathrm{mg}$ ascorbic acid, all the solids were dissolved and then the mixture was heated to $90{ }^{\circ} \mathrm{C}$. After $10 \mathrm{~min}$ at $90{ }^{\circ} \mathrm{C}, 1 \mathrm{~mL}$ of $3 \mathrm{mmol} / \mathrm{L} \mathrm{HAuCl}{ }_{4}$ solution $(5.1 \mathrm{mg}$ in $5 \mathrm{~mL}$ of water) was added as fast as possible under intense stirring. After that, the reaction took $3 \mathrm{~h}$ at $90^{\circ} \mathrm{C}$ and stirring. The obtained Au nanoparticles were washed with $100 \mathrm{~mL}$ of water and then suspended in ethanol [19].

\section{2. $\mathrm{Au} / \mathrm{CeO} \mathrm{O}_{2}$ Photocatalyst Synthesis}

$\mathrm{CeO}_{2}$ nanorods were synthesized with $\mathrm{Ce}\left(\mathrm{NO}_{3}\right)_{2} \cdot 6 \mathrm{H}_{2} \mathrm{O}$, sodium hydroxide and deionized water. A concentrated $\mathrm{NaOH}$ solution was prepared by adding $56 \mathrm{~g} \mathrm{NaOH}$ into $100 \mathrm{~mL}$ deionized water, the solution was cooled to ambient temperature before continuing. After that, $19.9 \mathrm{~g} \mathrm{Ce}\left(\mathrm{NO}_{3}\right)_{2} \cdot 6 \mathrm{H}_{2} \mathrm{O}$ was added to the $\mathrm{NaOH}$ solution and stirred for $30 \mathrm{~min}$, the resultant mixture was transferred into a $150 \mathrm{~mL}$ Teflon-lined autoclave and sealed. In order to produce nanorods, the autoclave was kept at $110^{\circ} \mathrm{C}$ for $24 \mathrm{~h}$. After that the solution obtained was washed with $1 \mathrm{~L}$ deionized water and $100 \mathrm{~mL}$ acetone and then transferred to a porcelain crucible, dried at $110^{\circ} \mathrm{C}$ for $6 \mathrm{~h}$ and calcined for $4 \mathrm{~h}$ at $400{ }^{\circ} \mathrm{C}$ [20]. Controlled morphology $\mathrm{CeO}_{2}$ was impregnated with $\mathrm{Au}$ nanoparticles using the incipient wetness method. After impregnation, the catalyst was dried at $90^{\circ} \mathrm{C}$ overnight and stored. 


\subsection{Catalyst Characterization}

Transmission electron microscopy (TEM) images were taken on a JEOL 2100 electron microscope (JEOL, Tokyo, Japan) operating at an accelerating voltage of $200 \mathrm{kV}$. The samples were prepared to TEM observations by dispersion in ethanol using an ultrasound bath and deposited onto holey-carbon copper grids. The solid-state UV-vis spectra were recorded on a Shimadzu UV-2600 PC spectrophotometer (Shimadzu Corporation, Kyoto, Japan). Wide-angle powder X-Ray Diffraction (XRD) data were recorded in a Rigaku Miniflex diffractometer (Rigaku Corporation, Tokyo, Japan) with $\mathrm{Cu} K \alpha$ radiation $(\lambda=1.54 \AA)$. The data of $2 \theta$ from 10 to $90^{\circ}$ were collected with a step size of $0.02^{\circ}$ and acquisition time of $1 \mathrm{~s}$. The diffraction patterns have been indexed by comparison with the Joint Committee on Powder Diffraction Standards (JCPDS) files. The concentrations of Au were determined by atomic absorption spectrometry (AA-Hitachi-Z8200 spectrometer, Hitachi, Japan). The surface area values were obtained by the BET method and the BJH model was used to determine the pore size distributions. The $\mathrm{N}_{2}$ adsorption and desorption measurements were carried out at $77 \mathrm{~K}$ using an Autosorb iQ2 Quantachrome Autosorb Equipment (Quantachrome, Boynton Beach, FL, USA).

\subsection{Photocatalytic Tests}

All the photocatalytic tests were conducted in a $100 \mathrm{~mL}$ pressurized photoreactor. In a typical photocatalytic experiment $2.5 \mathrm{~mL}$ mesitylene, $80 \mu \mathrm{L}$ benzyl alcohol, $100 \mu \mathrm{L}$ aniline, $50 \mathrm{mg}$ catalyst are mixed. With the reactor sealed to prevent solvent evaporation, the catalyst was suspended in an ultrasonic bath and then pressurized with 1 bar $\mathrm{O}_{2}$. Except when indicated, all the tests took $48 \mathrm{~h}$ at $50{ }^{\circ} \mathrm{C}$ with visible spectrum light $(300 \mathrm{~W})$ incidence under magnetic stirring. This temperature was chosen based on the best yields reported in the literature for this reaction [21]. The reaction products were analyzed by gas chromatography (GC). Two aliquots $(500 \mu \mathrm{L})$ were collected for each reactor batch, the first one before the $\mathrm{O}_{2}$ loading $(t=0)$ and the second one at the end of the reaction, typically $48 \mathrm{~h}$. In both cases, the catalyst was removed by centrifugation (11,000 rpm, $1 \mathrm{~min})$. Both samples were injected in a Shimadzu GC-2010 Plus gas chromatograph. The GC is equipped with a flame ionization detector, which enabled the separation and quantification of all reaction products using an RTX-Wax $(30 \mathrm{~m} \times 0.25 \mathrm{~mm} \times 0.25 \mu \mathrm{m})$ chromatographic column. The following temperature program was used during the analysis: $50^{\circ} \mathrm{C}$ for $3 \mathrm{~min}$; $15^{\circ} \mathrm{C} \mathrm{min}^{-1}$ until $220^{\circ} \mathrm{C}$.

\section{5. ${ }^{31} P$ NMR Measurement of TMP-Adsorbed Samples}

A sample of $200 \mathrm{mg}$ of $\mathrm{CeO}_{2}$ was placed in a glass tube and removed surface adsorbed water at $200{ }^{\circ} \mathrm{C}$ for $2 \mathrm{~h}$ under vacuum. After cooling down to room temperature, around $300 \mu \mathrm{mol} / \mathrm{catalyst} \mathrm{g}$ of trimethylphosphine (TMP) was then introduced. It was allowed around $15 \mathrm{~min}$ to reach the steady state for adsorption between TMP and catalyst surface. The sample was then vacuumed at room temperature for $5 \mathrm{~min}$ and thus excess non-adsorbed TMP molecules were removed gently without affecting all the chemisorbed peaks. The sample tube was then flame sealed for storage and transferred to Bruker $4 \mathrm{~mm} \mathrm{ZrO}_{2}$ rotor with a Kel-F endcap in a glove box under nitrogen atmosphere before NMR characterization. The solid state magic angle spinning (MAS) NMR experiments were carried out using a Bruker Avance III 400WB spectrometer at room temperature. The radiofrequency for decoupling was $59 \mathrm{kHz}$. The spectral width was $400 \mathrm{ppm}$, from 200 to $-200 \mathrm{ppm}$. The number of scannings was 800 . The ${ }^{31} \mathrm{P}$ chemical shifts were reported relative to $85 \%$ aqueous solution of $\mathrm{H}_{3} \mathrm{PO}_{4}$, with $\mathrm{NH}_{4} \mathrm{H}_{2} \mathrm{PO}_{4}$ as a secondary standard $(0.81 \mathrm{ppm})$.

\subsection{XPS Analysis}

Photoemission studies were conducted in a SPECSLAB II instrument equipped with a Ultra-high vacuum (UHV) chamber where the base pressure was less than $5 \times 10^{-9}$ Torr. The instrument has a hemispherical electron energy analyzer PHOIBOS-Has 3500150 with a 9-channels detector operating at $12 \mathrm{kV}$, pass energy (Epass) $=40 \mathrm{eV}, 0.2 \mathrm{eV}$ energy step and an $\mathrm{Al} \mathrm{K} \alpha \mathrm{X}$-ray source. The samples 
were placed on stainless steel sample-holders and were placed at the XPS prechamber and held there for $2 \mathrm{~h}$ in a vacuum. The collected spectra were adjusted with the CasaXPS 2.3.13 software. The C 1s peak was used as an internal standard for the charge correction (284.5 eV). A Shirley-type background was used [22]. The analysis of the Ce $3 \mathrm{~d}$ spectra was performed by adjusting 10 components, where 4 components correspond to the $\mathrm{Ce}^{3+}$ oxidation state $\left(\mathrm{u} 0, \mathrm{v} 0, \mathrm{u}^{\prime}, \mathrm{v}^{\prime}\right)$ and the 6 components correspond to the $\mathrm{Ce}^{4+}$ oxidation state $\left(\mathrm{u}, \mathrm{v}, \mathrm{u}^{\prime \prime}, \mathrm{v}^{\prime \prime}, \mathrm{u}^{\prime \prime \prime}, \mathrm{v}^{\prime \prime \prime}\right)$ [23]. The intensity ratio of $\mathrm{Ce} 3 \mathrm{~d}_{5 / 2} / \mathrm{Ce}$ $3 \mathrm{~d}_{3 / 2}$ doublets was set to 1.5 and the energy position was allowed to vary $\sim 2 \mathrm{eV}$ around the expected values [24], avoiding the inversion of positions.

\section{Results and Discussion}

The synthesis of $\mathrm{Au}$ NPs supported on the $\mathrm{CeO}_{2}$ nanorods $\left(\mathrm{Au} / \mathrm{CeO}_{2}\right)$ was performed by wet impregnation [20]. Figure 1A shows high-resolution transmission electron microscopy (HRTEM) images of the $\mathrm{Au} / \mathrm{CeO}_{2}$ nanorods. The $\mathrm{Au}$ NPs presented sizes in the 10-20 nm range. The $\mathrm{CeO}_{2}$ nanorods were around 10 and $200 \mathrm{~nm}$ in width and length, respectively (Figure S1). The Au loading as determined by atomic absorption spectroscopy corresponded to $0.64 \mathrm{wt} . \%$. X-ray diffractometry (Figure S2) showed only peaks assigned to the $\mathrm{CeO}_{2}$ phase. The UV-vis spectra of the $\mathrm{CeO}_{2}$ and $\mathrm{Au} / \mathrm{CeO}_{2}$ samples are depicted in Figure $1 \mathrm{~B}$ (black and red traces, respectively). It can be observed that $\mathrm{CeO}_{2}$ samples absorb light in the near-visible UV (<450 nm) [8]. Upon Au deposition, a band in the visible $(\approx 550 \mathrm{~nm})$ appears due to the LSPR dipolar mode in Au NPs $[8,12]$.
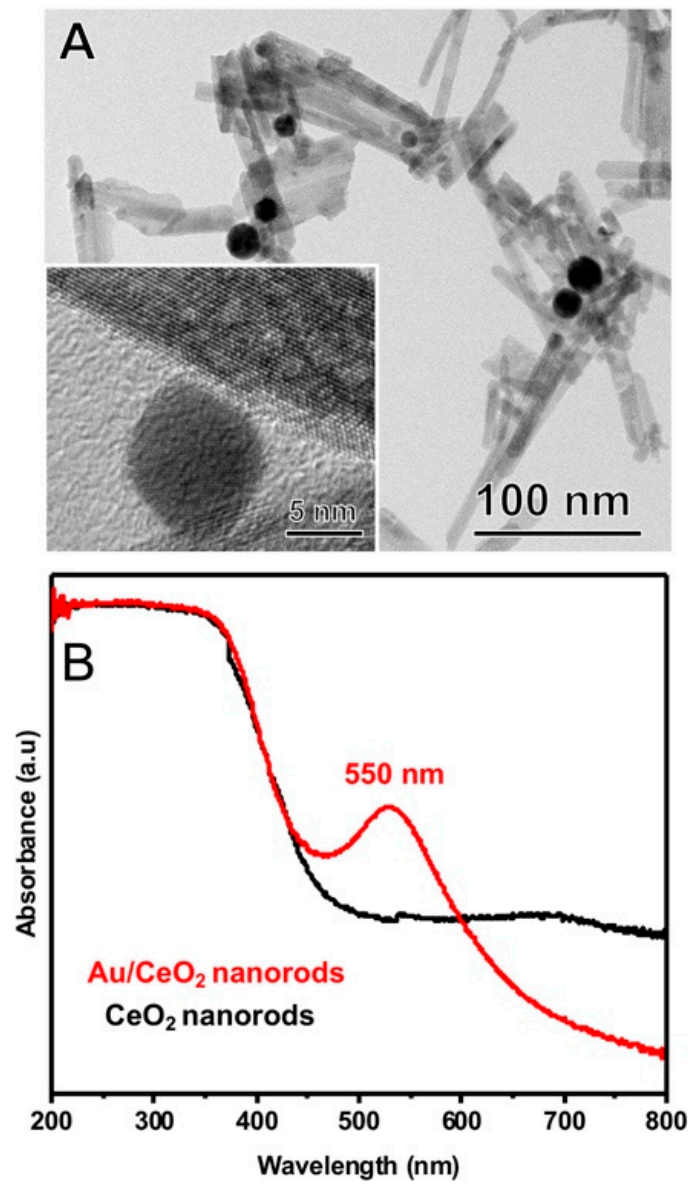

Figure 1. (A) HRTEM images for the $\mathrm{Au} / \mathrm{CeO}_{2}$ nanorods. (B) $\mathrm{UV}-$ vis extinction spectra for $\mathrm{CeO}_{2}$ and $\mathrm{Au} / \mathrm{CeO}_{2}$ nanorods (black and red traces, respectively).

Raman and XPS spectroscopies were employed to probe the metal-support interactions and oxygen vacancy properties in $\mathrm{CeO}_{2}$ and $\mathrm{Au} / \mathrm{CeO}_{2}$ nanomaterials. It is well-known that oxygen vacancies are 
structural defects that can adsorb $\mathrm{O}_{2}$ molecules and are correlated to oxygen mobility and storage capacity [25]. The Raman spectrum of $\mathrm{Au} / \mathrm{CeO}_{2}$ differed from the spectrum of $\mathrm{CeO}_{2}$ nanorods mainly by the main peak at $450-460 \mathrm{~cm}^{-1}$ assigned to oxygen ions vibrational mode in the $\mathrm{CeO}_{2}$ structure (Figure S3). This peak was strongly broadened in $\mathrm{Au} / \mathrm{CeO}_{2}$, which was caused by the smaller size of the crystalline domains due to the introduction of $\mathrm{Au}$ into the $\mathrm{CeO}_{2}$ structure. Besides, the Raman mode near $550 \mathrm{~cm}^{-1}$ and $600 \mathrm{~cm}^{-1}$ assigned to the presence of oxygen vacancies was more intense for $\mathrm{Au} / \mathrm{CeO}_{2}$ relative to the $\mathrm{CeO}_{2}$ nanorods, in agreement with the $\mathrm{Au}-\mathrm{CeO}_{2}$ metal-support interactions that increased the number of oxygen vacancy sites (Figure S3) [26,27]. This is further confirmed by the $\mathrm{Au} 4 \mathrm{f}$ core-level XPS for $\mathrm{Au} / \mathrm{CeO}_{2}$ nanorods (Figure S4). The $\mathrm{Au} 4 \mathrm{f}$ region showed two photoelectron peaks ascribed to $\mathrm{Au} 4 \mathrm{f}_{7 / 2}$ and $\mathrm{Au} 4 \mathrm{f}_{5 / 2}$ core-levels [12]. These peaks were shifted towards lower $\mathrm{BE}$ values, 82.7 and $86.3 \mathrm{eV}$, respectively, relative to $\mathrm{Au}$ in the metallic state [28]. The Ce $3 \mathrm{~d}$ core-level XPS spectra showed peak positions characteristic of $\mathrm{CeO}_{2}$ with the presence of $\mathrm{Ce}^{3+}$ in both $\mathrm{CeO}_{2}$ and $\mathrm{Au} / \mathrm{CeO}_{2}$ samples (Figure S5, Tables S1 and S2). The BET surface area for the samples $\mathrm{CeO}_{2}$ and $\mathrm{Au} / \mathrm{CeO}_{2}$ were $86.3 \mathrm{~m}^{2} \cdot \mathrm{g}^{-1}$ and $70.6 \mathrm{~m}^{2} \cdot \mathrm{g}^{-1}$ (Figure $\mathrm{S} 6$ and Table S3), respectively. The comparable values evidence that the $\mathrm{Au}$ deposition did not affect the $\mathrm{CeO}_{2}$ nanorods surface area. The same trend was observed for the pore diameter distribution (Figure S7 and Table S3).

We also studied the $\mathrm{Au} / \mathrm{CeO}_{2}$ and $\mathrm{CeO}_{2}$ nanorods by ${ }^{31} \mathrm{P}$-ssNMR analysis employing trimethylphosphine (TMP) as a surface probe. TMP is an electron donor molecule that can specifically form an adduct with surface Ce cation (i.e., TMP-Ce) [29]. In this case, the chemical shift of ${ }^{31} \mathrm{P}$ is expected to differentiate the strength of the adduct bond formed with various Ce acidities (or electron density) as the stronger surface Lewis acid site pushes $\delta^{31} \mathrm{P}$ to positive ppm [30]. As shown in Figure 2, the ${ }^{31} \mathrm{P}$ chemical shift of TMP-adsorbed nanorods shifted from 25.7 to $23.5 \mathrm{ppm}$ after Au incorporation, suggesting that the electron density of surface $\mathrm{Ce}$ was decreased upon contact with $\mathrm{Au}$. This lower electron density of surface $\mathrm{Ce}$ for the $\mathrm{Au} / \mathrm{CeO}_{2}$ nanorods sample might be associated with more oxygen vacancies and more active vacancies $[31,32]$.

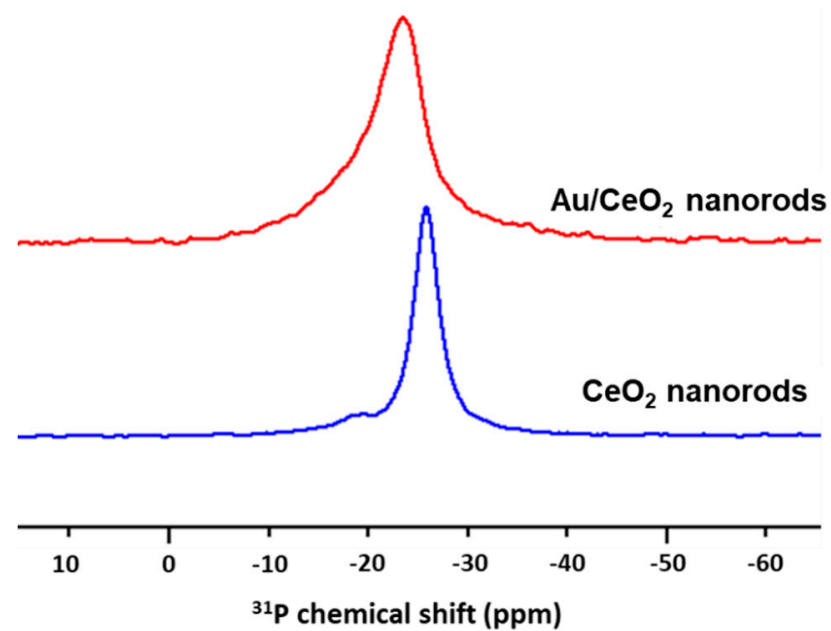

Figure 2. ${ }^{31} \mathrm{P}$ ss NMR spectra of $\mathrm{CeO}_{2}$ and $\mathrm{Au} / \mathrm{CeO}_{2}$ nanorods (blue and red traces, respectively) using trimethylphosphine (TMP) as a surface probe. After Au deposition, the ${ }^{31} \mathrm{P}$ NMR signal shifted from 25.7 to $23.5 \mathrm{ppm}$, suggesting that the electron density of the Ce surface is decreased upon Au deposition.

We then turned our attention to the catalytic activities towards the tandem imine production from alcohols and amines driven by light irradiation. We employed only benzyl alcohol and aniline as substrates [21]. It is accepted that this tandem reaction pathway starts with the alcohol oxidation to aldehyde followed by a spontaneous aldehyde-amine coupling, releasing the active redox site for a new reaction [6]. We employed a white lamp as the irradiation source and started by investigating the activities of the individual $\mathrm{Au} N \mathrm{NP}$ and $\mathrm{CeO}_{2}$ nanorods as catalysts as described in Table 1 . The use of $\mathrm{Au}$ NPs as catalysts showed a very low conversion percentage under light excitation (2.3\%, Entry 1), 
indicating that the LSPR excitation on Au NPs alone did not significantly contribute to the catalytic activity. The individual $\mathrm{CeO}_{2}$ nanorods (Table 1, Entries 2 and 3), on the other hand, displayed better activities, reaching around $62-63 \%$ conversion both in the dark and under light excitation. The higher activities occurred as a result of the presence of the oxygen vacancies, that acted as active sites for alcohol oxidation [8]. The similar activities detected for dark and light conditions show that the light excitation does not lead to any enhancements in catalytic activity in the $\mathrm{CeO}_{2}$ nanorods.

Table 1. Conversion and selectivity for the tandem conversion of benzyl alcohol into imines as a function of the catalysts and reaction conditions.

\begin{tabular}{|c|c|c|c|c|}
\hline \multirow{2}{*}{ Entry } & \multirow{2}{*}{ Catalyst } & \multirow{2}{*}{ Conversion of $\mathrm{BnOH}(\%)$} & \multicolumn{2}{|c|}{ Selectivity (\%) } \\
\hline & & & Imine & Other \\
\hline $1^{a}$ & $\begin{array}{c}\text { Au NPs } \\
\text { (Light) }\end{array}$ & 2.3 & 100 & 0 \\
\hline 2 & $\begin{array}{l}\text { Nanorods } \\
\text { (Light) }\end{array}$ & 62.9 & 96 & 4 \\
\hline 3 & $\begin{array}{l}\text { Nanorods } \\
\text { (No light) }\end{array}$ & 62.5 & 97 & 3 \\
\hline 4 & $\begin{array}{l}\mathrm{Au} / \text { nanorods } \\
\text { (Light) }\end{array}$ & 91.2 & 97 & 3 \\
\hline 5 & $\begin{array}{l}\text { Au/nanorods } \\
\text { (No light) }\end{array}$ & 69.5 & 97 & 3 \\
\hline $6^{\mathrm{d}}$ & $\begin{array}{c}\text { Au/nanorods } \\
\text { (Light, Ar atmosphere) }\end{array}$ & 31.7 & 97 & 3 \\
\hline $7^{d}$ & $\begin{array}{c}\text { Au/nanorods } \\
\text { (No light, Ar atmosphere) }\end{array}$ & 62.1 & 97 & 3 \\
\hline $8^{b}$ & $\begin{array}{c}\mathrm{Au} / \text { nanorods } \\
\text { (Light, electron scavenger) }\end{array}$ & 68.7 & 97 & 3 \\
\hline $9^{c}$ & $\begin{array}{c}\text { Au/nanorods } \\
\text { (Light, hole scavenger) }\end{array}$ & 30.7 & 97 & 3 \\
\hline
\end{tabular}

Typical experiment: $1 \mathrm{mmol}$ benzyl alcohol, $2 \mathrm{mmol}$ aniline, $50 \mathrm{mg}$ of catalyst, $1 \mathrm{bar}$ of $\mathrm{O}_{2}$, visible light, $50^{\circ} \mathrm{C}, 48 \mathrm{~h}$; a $\mathrm{Au}$ NPs colloidal suspension was added in equivalent amount to the $\mathrm{Au}$ concentration on $\mathrm{Au} / \mathrm{CeO}_{2}$ samples;

b $10 \mathrm{mmol}$ benzoquinone (electron scavenger); ${ }^{\mathrm{c}} 10 \mathrm{mmol}$ EDTA (hole scavenger); ${ }^{\mathrm{d}}$ Inert atmosphere (oxygen gas is replaced by argon).

Interestingly, the incorporation of $\mathrm{Au} N P$ s into the $\mathrm{CeO}_{2}$ nanorods $\left(\mathrm{Au} / \mathrm{CeO}_{2}\right)$ led to a significant increase in the catalytic activity under light excitation to $91 \%$, while keeping the selectivity to imines close to $97 \%$ (Table 1, Entry 4). In the dark, the activities corresponded to $69.5 \%$ (Table 1, Entry 5), which is close to the value detected for the individual $\mathrm{CeO}_{2}$ nanorods. The results from individual $\mathrm{Au}$ NPs (Entry 1) indicated that the enhancement observed for the $\mathrm{Au} / \mathrm{CeO}_{2}$ sample under light irradiation (Entry 4) was not only due to the presence of Au NPs and its plasmonic effects, for example. The observed effect was necessarily due to the synergism between $\mathrm{CeO}_{2}$ nanorods and the $\mathrm{Au} \mathrm{NPs}$, which was responsible for a conversion increment far beyond the sum of the conversion promoted by the $\mathrm{CeO}_{2}$ nanorods and the Au NPs individual counterparts.

We performed control experiments using electron or hole scavengers (Table 1, Entries 8 and 9) and by replacing the reaction medium with an Ar atmosphere (absence of $\mathrm{O}_{2}$ ) (Table 1, Entries 6 and 7) to better understand the photocatalytic enhancements in $\mathrm{Au} / \mathrm{CeO}_{2}$ nanorods. Under light excitation, the use of an Ar atmosphere decreased the conversion to 31.7\% (Entry 6) relative to $91.2 \%$ in $\mathrm{O}_{2}$ (Entry 4). This demonstrates that the $\mathrm{O}_{2}$ plays a central role in this conversion driven by light. Suppression of catalytic activity was observed under the Ar atmosphere and light excitation relative to the utilization 
of the Ar atmosphere and dark conditions (62.1\%, Entry 7). Surprisingly, under dark conditions, the conversion in the $\mathrm{O}_{2}$ and Ar atmosphere was similar (Entries 5 and 7, respectively). This result demonstrates that the presence of $\mathrm{O}_{2}$ and the plasmonic excitation (light irradiation) are required for the high activities detected for the $\mathrm{Au} / \mathrm{CeO}_{2}$ nanorods (Entry 4).

Based on these results we would like to propose that the unique structure of the (110) surface facets in the $\mathrm{CeO}_{2}$ nanorods (Figure 3A) allow first the adsorption of benzyl alcohol (via a deprotonation pathway) (Figure 3B). As Tamura and collaborators demonstrated, this deprotonation step does not depend on $\mathrm{O}_{2}$ [6] and is easily accomplished by the Lewis acid (LA) sites on the $\mathrm{CeO}_{2}$ (110) surface facet (i.e., $\mathrm{Ce}^{4+}$ surficial species) $[29,33,34]$. Even under dark conditions some synergistic effect is observed for the $\mathrm{Au} / \mathrm{CeO}_{2}$, the Au NPs promotes an increment from $62.5 \%$ (Entry 3) to $69.5 \%$ (Entry 5) for the imine formation. It is well-established for alcohol oxidation investigations (not light-driven) that this synergistic effect occurs due to the charge separation in the interface $\mathrm{Au} / \mathrm{CeO}_{2}$, in which the $\mathrm{Au}$ NPs gives an $\mathrm{e}^{-}$for the $\mathrm{Ce}^{4+}$ reducing it to $\mathrm{Ce}^{3+}$ and the Au NPs keep the positive charge $[33,35]$. These positive-charged Au species are responsible for the C-H activation, which is the limiting step of the reaction $[33,35]$. Under light irradiation, this synergistic effect, due to the charge separation, is further promoted. Upon LSPR excitation of the Au NPs, hot electrons and hot holes are generated $[13,14]$. The hot electrons can be trapped at the redox sites of the $\mathrm{CeO}_{2}$ while the hot holes remain at the $\mathrm{Au}$ NPs (Figure $3 \mathrm{C}$ and Figure S8). The electrons in the $\mathrm{CeO}_{2}$ will reduce some $\mathrm{Ce}^{4+}$ into $\mathrm{Ce}^{3+}$ generating vacancies (Figure 3C). In the next step, hot holes on Au NPs can contribute to the $\alpha-\mathrm{H}$ abstraction from the adsorbed benzyl alcohol substrate leading to a carbon-centered radical (Figure 3C,D) and $\mathrm{H}-\mathrm{Au}$ species over the Au NP [35-37]. These H-Au species were trapped by 5,5-dimethyl-1-pyrroline N-oxide (DMPO) in electron paramagnetic resonance (EPR) experiments reported by Garcia et al. under similar conditions [35]. Simultaneously, trapped hot electrons can be transferred to $\mathrm{O}_{2}$ species adsorbed on vacancies to give surface-bound $\bullet_{2}{ }^{-}$(Figure 3C,D). We then hypothesize that the carbon-centered radical can recombine with the electrons trapped on the vacancies $\left(\mathrm{Ce}^{3+}\right.$ species) regenerating the $\mathrm{Ce}^{4+}$ and forming benzaldehyde, which couples with the aniline enabling the tandem formation of the imines. The cerium-coordinated superoxide species have the important role of regenerating the surface of Au NPs by hydrogen abstraction from $\mathrm{H}-\mathrm{Au}$ (Figure 3E), leading to $\mathrm{H}_{2} \mathrm{O}_{2}$ [33]. By replacing $\mathrm{O}_{2}$ with an inert atmosphere, there is a decrease in the conversion percentage as no $\mathrm{O}_{2}$ is available to regenerate the Au surface. The deactivation of the Au surface in the absence of an oxidant can be noticed by comparing the reactions for the $\mathrm{Au} /$ nanorods sample under $\mathrm{Ar}$ (Entry 7) and nanorods (no Au) without irradiation under $\mathrm{O}_{2}$ (Entry 2), both display similar activities (62.1\% and $62.9 \%$, respectively), which are lower than the $\mathrm{Au} /$ nanorods sample under $\mathrm{O}_{2}$ (Entry 7). Garcia et al. reported that this reaction can be performed in the absence of $\mathrm{O}_{2}$ when DMPO is used to abstract the $\mathrm{H}$-Au species and regenerate the $\mathrm{Au}$ NPs [35]. Therefore, it is plausible that in the absence of an oxidant, chemisorbed hydrogens stay bonded to the Au NPs surface, poisoning its surface. This is supported by the chromatogram for the reaction products in the absence of $\mathrm{O}_{2}$, which displayed the same profile and no amines were detected.

In the absence of $\mathrm{O}_{2}$ and under light the catalyst activity is suppressed, reaching smaller values relative to the reaction in the dark (both in the presence of $\mathrm{O}_{2}$ and Ar atmosphere). It is plausible that hot electrons trapped at the oxygen vacancy sites are not transferred to adsorbed $\mathrm{O}_{2}$. Instead, they can be transferred to the adsorbed benzyl alcohol species disfavoring the $\alpha-\mathrm{H}$ abstraction and suppressing the reaction rates. Under dark conditions, the LSPR excitation does not play a role in this transformation and activities under $\mathrm{Ar}$ and $\mathrm{O}_{2}$ yield similar results. Thus, in the absence of light excitation, the $\mathrm{O}_{2}$ plays an even more important role over the benzyl alcohol oxidation.

This proposed reaction pathway is also supported by the conversion percentage for the $\mathrm{Au} / \mathrm{CeO}$ nanorods in the presence of electrons or hole scavengers (under light excitation and $\mathrm{O}_{2}$ atmosphere). In the presence of electron scavengers, adsorbed $\mathrm{O}_{2}$ species activation is disfavored and the resulting activities are similar for the $\mathrm{Au} / \mathrm{CeO}_{2}$ nanorods under dark conditions and $\mathrm{CeO}_{2}$ nanorods. In this case, activities are dictated by the $\mathrm{CeO}_{2}$ redox sites. In the presence of hole scavengers, the $\alpha-\mathrm{H}$ abstraction from the adsorbed benzyl alcohol substrate is suppressed, and conversion percentages decrease to 
30.7\%. Hole scavengers can deactivate the positive charged $\mathrm{Au}$ species as well as the $\mathrm{CeO}_{2}$ redox sites, both active sites present in the catalyst. This result suggests that the synergistic effect is taking place through a charge separation process and not only by a heating effect promoted by the Au NPs. The possibility of a reaction between EDTA (hole scavenger) and the reactants was excluded by the absence of new peaks in the GC-MS analysis. Under Ar atmosphere, activity suppression is also observed, this effect is related to the deactivation of the Au surface, once $\bullet \mathrm{O}_{2}{ }^{-}$formation is avoided and the catalyst surface is not regenerated. Furthermore, the absence of $\mathrm{O}_{2}$ or electron receptor species might influence the $\mathrm{Ce}^{3+} / \mathrm{Ce}^{4+}$ ratio, leading to a deactivation of the redox sites of the $\mathrm{CeO}_{2}[6]$.

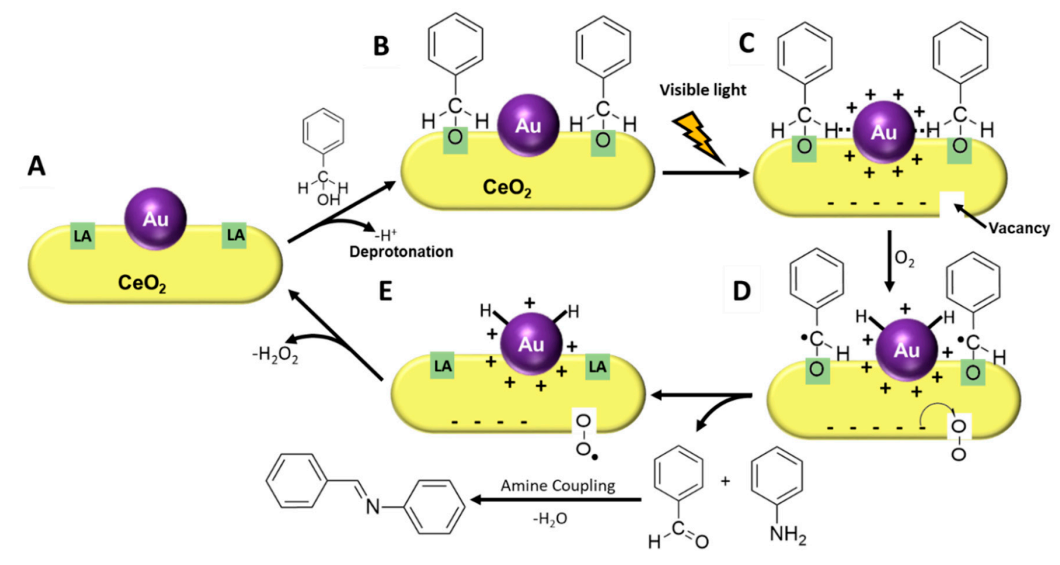

Figure 3. Schematic representation of the proposed photocatalytic mechanism. The LA sites (A) enable the adsorption of benzyl alcohol (B), via a deprotonation pathway. Localized surface plasmon resonance (LSPR) excitation leads to the formation of hot electrons and holes (C). Hot electrons get trapped at vacancy sites, where they activate adsorbed $\mathrm{O}_{2}(\mathbf{C}, \mathbf{D})$. Hot holes facilitate the $\alpha-\mathrm{H}$ abstraction from the adsorbed benzyl alcohol (C,D). The cerium-coordinated superoxide species can abstract hydrogen from $\mathrm{H}$-Au species (E), regenerating the catalyst surface. The formed benzaldehyde couples with aniline in the next step to generate the imine.

\section{Conclusions}

In summary, we have demonstrated that the plasmonic excitation in $\mathrm{Au} / \mathrm{CeO}_{2}$ nanorods enables an alternative reaction pathway that leads to improved catalytic performance relative to individual $\mathrm{CeO}_{2}$ nanorods towards the visible-light-driven tandem synthesis of imines via alcohol oxidation. Our results demonstrated that the presence of $\mathrm{O}_{2}$, oxygen vacancies in $\mathrm{CeO}_{2}$, and both hot electrons and holes generated as a result of LSPR excitation in the visible range were crucial to the detected photocatalytic performances. While the hot electrons lead to the formation of oxygen vacancies, enabling the adsorption of $\mathrm{O}_{2}$, hot holes generate positive-charged Au species, which facilitate the $\alpha$-H abstraction from the adsorbed benzyl alcohol. The produced carbon-centered radical can recombine with the electrons trapped in the vacancies producing the benzaldehyde, which couples with the aniline, enabling the tandem formation of the imines. The cerium-coordinated superoxide regenerates the surface of $\mathrm{Au}$ NPs by hydrogen abstraction, forming $\mathrm{H}_{2} \mathrm{O}_{2}$. We believe the results presented herein can inspire the development of photocatalysts containing plasmonic components that can enable improved performance and selectivity via reaction pathways that are not accessible in semiconducting NPs or external heating.

Supplementary Materials: The following are available online at http://www.mdpi.com/2079-4991/10/8/1530/s1, Figure S1: $\mathrm{TEM} \mathrm{CeO}_{2}$ nanorods, Figure S2: XRD, Figure S3: Raman spectra, Figure S4 and S5: XPS spectra, Table S1: Binding energies Au 4f XPS, Table S2: Binding energies O 1s XPS, Figure S6: Isotherms BET, Figure S7: $\mathrm{BJH}$ pore diameter distribution, Table S3: BET surface area and average pore diameter, Figure S8: Scheme of the energy level diagram for the $\mathrm{Au} / \mathrm{CeO}_{2}$ nanorod photocatalyst.

Author Contributions: Conceptualization, I.F.T., R.S.G. and P.H.C.C.; methodology, I.F.T., R.S.G., M.S.H. and P.H.C.C.; XPS analysis, J.Q.; BET analysis, I.F.S.; ${ }^{31}$ P NMR, Y.-K.P.; investigation, I.F.T., M.S.H., R.S.G., G.F.S.R.R.,Y.-K.P., I.F.S. J.Q. and P.H.C.C.; resources, I.F.T., M.S.H., R.S.G., G.F.S.R.R.,Y.-K.P., I.F.S. J.Q. and P.H.C.C.; 
data curation, I.F.T. and M.S.H.; writing-original draft preparation, I.F.T. and P.H.C.C.; writing-review and editing, I.F.T., I.F.S. and P.H.C.C.; supervision, I.F.T. and P.H.C.C.; project administration, P.H.C.C.; funding acquisition, P.H.C.C. All authors have read and agreed to the published version of the manuscript.

Funding: This research was funded by CAPES, CNPq and FAPESP (grant numbers 423196/2018-9 and 2017/50118-9).

Acknowledgments: P.H.C.C. thanks the Jane and Aatos Erkko Foundation and the University of Helsinki for financial support.

Conflicts of Interest: The authors declare no conflict of interest.

\section{References}

1. Wang, F.; Li, Q.; Xu, D. Recent Progress in Semiconductor-Based Nanocomposite Photocatalysts for Solar-to-Chemical Energy Conversion. Adv. Energy Mater. 2017, 7, 1700529. [CrossRef]

2. Parsons, P.J.; Penkett, C.S.; Shell, A.J. Tandem reactions in organic synthesis: Novel strategies for natural product elaboration and the development of new synthetic methodology. Chem. Rev. 1996, 96, 195-206. [CrossRef]

3. Anastas, P.T.; Warner, J.C. Principles of green chemistry. In Green Chemistry Theory and Practice; Oxford University Press: New York, NY, USA, 1998; pp. 29-56.

4. Behr, A.; Vorholt, A.J.; Ostrowski, K.A.; Seidensticker, T. Towards resource efficient chemistry: Tandem reactions with renewables. Green Chem. 2014, 16, 982-1006. [CrossRef]

5. Patil, R.D.; Adimurthy, S. Catalytic methods for imine synthesis. Asian J. Org. Chem. 2013, 2, 726-744. [CrossRef]

6. Tamura, M.; Tomishige, K. Redox properties of $\mathrm{CeO}_{2}$ at low temperature: The direct synthesis of imines from alcohol and amine. Angew. Chem. Int. Ed. 2015, 54, 864-867. [CrossRef]

7. Zhang, J.; Yang, J.; Wang, J.; Ding, H.; Liu, Q.; Schubert, U.; Rui, Y.; Xu, J. Surface oxygen vacancies dominated $\mathrm{CeO}_{2}$ as efficient catalyst for imine synthesis: Influences of different cerium precursors. Mol. Catal. 2017, 443, 131-138. [CrossRef]

8. Montini, T.; Melchionna, M.; Monai, M.; Fornasiero, P. Fundamentals and catalytic applications of $\mathrm{CeO}_{2}$-based materials. Chem. Rev. 2016, 116, 5987-6041. [CrossRef]

9. Araújo, T.P.; Quiroz, J.; Barbosa, E.M.; Camargo, P.H.C. Understanding plasmonic catalysis with controlled nanomaterials based on catalytic and plasmonic metals. Curr. Opin. Colloid Interface Sci. 2019, 39, 110-122.

10. Wang, J.; Ando, R.A.; Camargo, P.H.C. Controlling the Selectivity of the Surface Plasmon Resonance Mediated Oxidation of p-Aminothiophenol on Au Nanoparticles by Charge Transfer from UV-excited $\mathrm{TiO}_{2}$. Angew. Chem. Int. Ed. 2015, 54, 6909-6912. [CrossRef]

11. Rodrigues, T.S.; da Silva, A.G.M.; Camargo, P.H.C. Nanocatalysis by noble metal nanoparticles: Controlled synthesis for the optimization and understanding of activities. J. Mater. Chem. A 2019, 7, 5857-5874. [CrossRef]

12. Geonmonond, R.S.; Quiroz, J.; Rocha, G.F.S.R.; Oropeza, F.E.; Rangel, C.J.; Rodrigues, T.S.; Hofmann, J.P.; Hensen, E.J.M.; Ando, R.A.; Camargo, P.H.C. Marrying SPR excitation and metal-support interactions: Unravelling the contribution of active surface species in plasmonic catalysis. Nanoscale 2018, 10, 8560-8568. [CrossRef]

13. Zhu, K.; Wang, C.; Camargo, P.H.C.; Wang, J. Investigating the effect of $\mathrm{MnO}_{2}$ band gap in hybrid $\mathrm{MnO}_{2}-\mathrm{Au}$ materials over the SPR-mediated activities under visible light. J. Mater. Chem. A 2019, 7, 925-931. [CrossRef]

14. Wang, J.; de Freitas, I.C.; Alves, T.V.; Ando, R.A.; Fang, Z.; Camargo, P.H.C. On the effect of native $\mathrm{SiO}_{2}$ on Si over the SPR-mediated photocatalytic activities of Au and Ag nanoparticles. Chem. Eur. J. 2017, 23, 7185-7190. [CrossRef]

15. Haruta, M.; Daté, M. Advances in the catalysis of Au nanoparticles. Appl. Catal. A Gen. 2001, 222, 427-437. [CrossRef]

16. Coquet, R.; Howard, K.L.; Willock, D.J. Theory and simulation in heterogeneous gold catalysis. Chem. Soc. Rev. 2008, 37, 2046-2076. [CrossRef]

17. Dhakshinamoorthy, A.; Asiri, A.M.; Garcia,H. Metal organic frameworks as versatile hosts of Au nanoparticles in heterogeneous catalysis. ACS Catal. 2017, 7, 2896-2919. [CrossRef]

18. Shahzad, S.A.; Sajid, M.A.; Khan, Z.A.; Canseco-Gonzalez, D. Gold catalysis in organic transformations: A review. Synth. Commun. 2017, 47, 735-755. [CrossRef] 
19. Damato, T.C.; de Oliveira, C.C.S.; Ando, R.M.A.; Camargo, P.H.C. A facile approach to $\mathrm{TiO}_{2}$ colloidal spheres decorated with Au nanoparticles displaying well-defined sizes and uniform dispersion. Langmuir 2013, 29, 1642-1649. [CrossRef]

20. Si, R.; Flytzani-Stephanopoulos, M. Shape and crystal-plane effects of nanoscale ceria on the activity of $\mathrm{Au}-\mathrm{CeO}_{2}$ catalysts for the water-gas shift reaction. Angew. Chem. Int. Ed. 2008, 47, 2884-2887. [CrossRef]

21. Zhang, Z.; Wang, Y.; Wang, M.; Lü, J.; Li, L.; Zhang, Z.; Li, M.; Jiang, J.; Wang, F. An investigation of the effects of $\mathrm{CeO}_{2}$ crystal planes on the aerobic oxidative synthesis of imines from alcohols and amines. Chin. J. Catal. 2015, 36, 1623-1630. [CrossRef]

22. Shirley, D.A. High-resolution X-ray photoemission spectrum of the valence bands of gold. Phys. Rev. B 1972, 5, 4709. [CrossRef]

23. Burroughs, P.; Hamnett, A.; Orchard, A.F.; Thornton, G. Satellite structure in the X-ray photoelectron spectra of some binary and mixed oxides of lanthanum and cerium. J. Chem. Soc. Dalton Trans. 1976, 1686-1698. [CrossRef]

24. Maslakov, K.I.; Teterin, Y.A.; Popel, A.J.; Teterin, A.Y.; Ivanov, K.E.; Kalmykov, S.N.; Petrov, V.G.; Petrov, P.K.; Farnan, I. XPS study of ion irradiated and unirradiated $\mathrm{CeO}_{2}$ bulk and thin film samples. Appl. Surf. Sci. 2018, 448, 154-162. [CrossRef]

25. Zhao, K.; Qi, J.; Yin, H.; Wang, Z.; Zhao, S.; Ma, X.; Wan, J.; Chang, L.; Gao, Y.; Yu, R. Efficient water oxidation under visible light by tuning surface defects on ceria nanorods. J. Mater. Chem. A 2015, 3, 20465-20470. [CrossRef]

26. Li, J.; Liu, X.; Zhan, W.; Guo, Y.; Guo, Y.; Lu, G. Preparation of high oxygen storage capacity and thermally stable ceria-zirconia solid solution. Catal. Sci. Technol. 2016, 6, 897-907. [CrossRef]

27. Kalamaras, C.M.; Petallidou, K.C.; Efstathiou, A.M. The effect of $\mathrm{La}^{3+}$-doping of $\mathrm{CeO}_{2}$ support on the water-gas shift reaction mechanism and kinetics over $\mathrm{Pt} / \mathrm{Ce}_{1-\mathrm{x}} \mathrm{La}_{\mathrm{x}} \mathrm{O}_{2-\delta}$. Appl. Catal. B Environ. 2013, 136, 225-238. [CrossRef]

28. Casaletto, M.P.; Longo, A.; Martorana, A.; Prestianni, A.; Venezia, A.M. XPS study of supported gold catalysts: The role of $\mathrm{Au}^{0}$ and $\mathrm{Au}^{+\delta}$ species as active sites. Surf. Interface Anal. 2006, 38, 215-218. [CrossRef]

29. Tan, Z.; Li, G.; Chou, H.-L.; Li, Y.; Yi, X.; Mahadi, A.H.; Zheng, A.; Tsang, S.C.E.; Peng, Y.-K. Differentiating Surface Ce Species Among $\mathrm{CeO}_{2}$ Facets by Solid-State NMR for Catalytic Correlation. ACS Catal. 2020, 10, 4003-4011. [CrossRef]

30. Zheng, A.; Liu, S.-B.; Deng, F. ${ }^{31}$ P NMR chemical shifts of phosphorus probes as reliable and practical acidity scales for solid and liquid catalysts. Chem. Rev. 2017, 117, 12475-12531. [CrossRef]

31. Kim, H.Y.; Lee, H.M.; Henkelman, G. $\mathrm{CO}$ oxidation mechanism on $\mathrm{CeO}_{2}$-supported Au nanoparticles. J. Am. Chem. Soc. 2012, 134, 1560-1570. [CrossRef] [PubMed]

32. Song, W.; Hensen, E.J.M. Mechanistic aspects of the water-gas shift reaction on isolated and clustered Au atoms on $\mathrm{CeO}_{2}$ (110): A density functional theory study. ACS Catal. 2014, 4, 1885-1892. [CrossRef]

33. Abad, A.; Concepción, P.; Corma, A.; García, H. A collaborative effect between gold and a support induces the selective oxidation of alcohols. Angew. Chem. Int. Ed. 2005, 44, 4066-4069. [CrossRef] [PubMed]

34. Tamura, M.; Tomishige, K. Scope and reaction mechanism of $\mathrm{CeO}_{2}$-catalyzed one-pot imine synthesis from alcohols and amines. J. Catal. 2020, 389, 285-296. [CrossRef]

35. Maldotti, A.; Molinari, A.; Juárez, R.; Garcia, H. Photoinduced reactivity of Au-H intermediates in alcohol oxidation by gold nanoparticles supported on ceria. Chem. Sci. 2011, 2, 1831-1834. [CrossRef]

36. Conte, M.; Miyamura, H.; Kobayashi, S.; Chechik, V. Spin trapping of Au-H intermediate in the alcohol oxidation by supported and unsupported gold catalysts. J. Am. Chem. Soc. 2009, 131, 7189-7196. [CrossRef]

37. Li, H.; Qin, F.; Yang, Z.; Cui, X.; Wang, J.; Zhang, L. New reaction pathway induced by plasmon for selective benzyl alcohol oxidation on $\mathrm{BiOCl}$ possessing oxygen vacancies. J. Am. Chem. Soc. 2017, 139, 3513-3521. [CrossRef]

(C) 2020 by the authors. Licensee MDPI, Basel, Switzerland. This article is an open access article distributed under the terms and conditions of the Creative Commons Attribution (CC BY) license (http://creativecommons.org/licenses/by/4.0/). 